\title{
A Cockpit-based Application for Traffic Aware Trajectory Optimization
}

\author{
Sharon E. Woods", Robert A. Vivona ${ }^{\dagger}$, David A. Roscoe \\ Engility Corporation, Billerica, MA, 01821 \\ and \\ David J. Wing** and Mark G. Ballin ${ }^{\dagger \dagger}$ \\ NASA Langley Research Center, Hampton, VA, 23681
}

\begin{abstract}
The Traffic Aware Planner (TAP) is a cockpit-based advisory tool designed to be hosted on a Class 2 Electronic Flight Bag and developed to enable the concept of Traffic Aware Strategic Aircrew Requests (TASAR). This near-term concept provides pilots with optimized route changes that reduce fuel burn or flight time, avoids interactions with known traffic, weather and restricted airspace, and may be used by the pilots to request a trajectory change from air traffic control. TAP's internal architecture and algorithms are derived from the Autonomous Operations Planner, a flight-deck automation system developed by NASA to support research into aircraft self-separation. This paper reviews the architecture, functionality and operation of TAP.
\end{abstract}

\section{Introduction}

$\mathrm{O}$ PTIMIZATION of aircraft trajectories to minimize flight time and/or fuel burn is an attractive initiative of commercial aircraft operations but is currently constrained in the current National Airspace System (NAS). Aircraft operating under Instrument Flight Rules (IFR) fly on trajectories approved by Air Traffic Control (ATC). To make changes to their trajectory for any (non-emergency) reason, aircrews must request the change from ATC and obtain approval before changing their current route. When constructing these trajectory change requests, aircrews do not have the same knowledge as ATC about surrounding traffic, and the request may subsequently be rejected by ATC due to a conflict. Aircrews may also not be aware of optimization opportunities because there is not an existing tool actively seeking them out.

With the adoption of Automatic Dependent Surveillance - Broadcast (ADS-B) in the NAS, an opportunity exists to give aircrews the ability to construct optimizations that account for surrounding traffic that may improve their chances of being approved by ATC. A flight-deck system designed to incorporate traffic awareness data from ADSB IN, along with avionics data such as current flight plan and state, could provide the flight crew with suggested optimizations of their route that would not be rejected by ATC due to conflicts with surrounding traffic. Additional flight-deck data sources could further be utilized by such a system to provide optimizations that account for updated winds, convective weather, special use airspace, airspace boundaries, and terrain.

The Traffic Aware Planner (TAP), developed under contract to NASA Langley Research Center, is a prototype optimization tool designed to test the concept of using ADS-B data and algorithms from previous research of airborne conflict detection and resolution to provide aircrews with conflict-free optimization suggestions. Designed to support research of the concept of Traffic Aware Strategic Aircrew Requests (TASAR) ${ }^{1,2}$, the tool is advisoryonly, operates on a Class 2 Electronic Flight Bag (EFB), and will be flight tested in the NAS on a research aircraft in the Fall of 2013.

* Principal Software Engineer, 300 Concord Road, Suite 400, AIAA Member

${ }^{\dagger}$ Chief Research Engineer, 300 Concord Road, Suite 400, AIAA Associate Fellow

* Senior Software Engineer, 300 Concord Road, Suite 400, AIAA Member

$\S$ Software Engineer, 300 Concord Road, Suite 400

${ }^{* *}$ Principal ATM Research Engineer, NASA LaRC, Mail Stop 152, AIAA Member

${ }^{\dagger}$ Project Scientist, NASA NextGen Concepts and Technology Development Project, NASA LaRC, Mail Stop 156A, AIAA Associate Fellow 
This paper will discuss the motivation for developing a flight-deck optimization tool and give an overview of the concept of TASAR, including scenarios on how such a tool may be used. The operational capabilities and functionality of TAP will be discussed, as well as an overview of future developments that may be considered.

\section{Motivation}

An on-board flight optimization tool has a number of advantages over the current means of flight optimization typically used in the NAS. Most current flight optimization happens pre-departure, when a flight plan is determined based on available observations and forecasts of winds and weather, the optimal performance of the aircraft type, and ATC procedures for filing flight plans. After departure, further flight optimization from dispatchers is dependent on their available workload and motivation to seek optimization opportunities for individual flights. Pilots, who have dedicated attention to their own flight, do not currently have tools that monitor for optimization opportunities with updated weather, winds, and traffic while en-route. Their requests to ATC for flight optimization are largely based on their past experience and knowledge of a given route and are made without sufficient knowledge of surrounding traffic that may cause ATC to deny the request. Opportunities for optimization based on updated data such as favorable winds are lost due to the pilot not having a means to view or analyze the data. Also, visualizing opportunities for optimization become increasingly complex the more factors are considered. Automated monitoring of such opportunities allow for constant analysis that can be utilized when it provides the maximum benefit.

TAP provides both automated and manual support to pilots for flight optimization, leveraging its access to onboard data of the aircraft's current state and route, surrounding traffic, weather, winds, temperature, and restricted airspace. TAP is designed specifically to be a support tool, and its use does not change any of the current procedures for requesting route changes from ATC or implementing them within the cockpit.

An additional motivation for the development of TAP was that it could provide aircraft operators a near-term benefit from ADS-B IN equipage. ${ }^{3}$ Although the Federal Aviation Administration (FAA) is due to benefit from increased surveillance capabilities and reduced radar costs from their mandate that all aircraft broadcast position data over ADS-B by 2020, users have not yet seen a measurable benefit to offset the cost of the equipment. One factor in users not seeing a significant benefit from ADS-B adoption is the lack of on-board applications that utilize ADS-B IN data. TAP has been designed to operate in a current-day cockpit environment and provide immediate benefits to each individual aircraft regardless of whether other users are equipped with TAP or even ADS-B OUT. As more aircraft adopt ADS-B OUT, benefits of TAP usage will increase as its traffic awareness increases. By providing immediate benefits of ADS-B IN on a per-aircraft basis, an acceleration of overall ADS-B equipage by the user community may occur.

\section{Concept and Definitions}

\section{A. TASAR Concept}

The TASAR concept ${ }^{1,2}$ proposes to use on-board data and decision-support automation to provide pilots with an optimization replanning tool that will result in route change requests which are more likely to be approved by ATC. Improving the chance of pilot requests being approved can potentially reduce workload on air traffic control without changing current procedures. Automation provides algorithms and data processing capabilities that allow pilot awareness of optimization opportunities that may have been previously obscured. Initial benefits analysis of TAS AR found that aircraft equipped with TASAR capabilities can reduce flight travel time from one to four minutes and fuel burn by 50 to 550 pounds. $^{4}$

TAP is a prototype automation designed to enable TASAR operations. For the purposes of describing TAP, the following key aspects of the "Traffic Aware Strategic Aircrew Requests" concept are highlighted:

1) Traffic Aware: TAP assumes that the ownship aircraft is equipped with ADS-B receivers which will supply it with timely and accurate traffic surveillance of nearby aircraft. TAP was also designed to accept data from potential sources of traffic data via data link or internet access. Although benefits can accrue without traffic awareness, greater benefits are expected when traffic is considered.

2) Strategic: TAP assumes that the ownship aircraft has a Flight Management System (FMS) that will allow them to enter "strategic" (i.e., trajectory based) route changes. Strategic also refers to the proactive and informed nature of this flight-optimization concept.

3) Aircrew Requests: TAP is a cockpit-based tool that leverages flight-specific avionics data, pilot availability during low-workload operations, and the ability to formulate and issue timely ATC requests. TAP is an advisory tool and does not change the current NAS procedures. Once the pilot has 
used TAP to determine a route change, the request to ATC follows the same procedures that exist today in the NAS.

\section{B. Optimization}

TAP is a flight optimization tool, and its main objective is to assist aircrews in finding route changes that will optimize parameters such as fuel-burn and flight time that are also conflict-free and therefore more approvable by ATC. TAP's architecture and algorithms were derived from the NASA Autonomous Operations Planner (AOP).$^{5-8}$ AOP is a research tool for investigating airborne self-separation, and its main objective is to detect and resolve conflicts. TAP utilizes AOP's conflict detection and resolution capabilities to assure that its optimizations are conflict-free, but its main goal is to optimize the current route, not to detect and resolve conflicts on the current route.

\section{AOP-Related Definitions}

Since TAP was derived from AOP's architecture and algorithms, descriptions of its capabilities use several terms in common with those of AOP: ${ }^{5}$

1) Ownship: Refers to the aircraft onboard which TAP is installed.

2) State: The state of an ownship aircraft is the instantaneous snapshot of known avionics data that TAP is aware of when performing optimizations. State data may include (depending on the ownship aircraft's avionics data feed capabilities) current pressure altitude, position, airspeed, groundspeed, heading and track angles, fuel burn, and weight.

3) Active Route: The active route is the route the ownship aircraft is currently navigating with active lateral navigation (LNAV) and vertical navigation (VNAV) guidance. TAP's optimization solutions consist of proposed modifications to this active route.

4) Hazards: There are two classes of hazards: Traffic Hazards, which are all aircraft other than the ownship aircraft, and Area Hazards, which are four-dimensional regions of unusable airspace due to convective weather, special use airspace (SUA), terrain, or other ATC restrictions.

5) Conflict: A conflict is a predicted loss of the minimum required separation between a TAP-predicted four-dimensional (4D) trajectory and a hazard. To increase the chances of ATC approval of aircrew requests,TAP will apply a buffer to the formal separation standards for traffic.

\section{Outcomes}

For each route change TAP suggests, it calculates how much fuel and flight time the new route will take, based on the predicted 4D trajectory. These values will be compared to the fuel and flight time of the active route's predicted 4D trajectory to calculate the outcomes of the suggested route change in terms of the predicted change in travel time and pounds of fuel consumed. Outcome is also used to refer to the conflict detection results of TAP's evaluations.

\section{E. TASAR Scenarios}

The following three scenarios provide examples of situations where TAP could provide flight optimization benefits. They do not cover all the capabilities of TAP, but they give a general overview of how TAP could improve the existing flight optimization process.

\section{Automated Monitoring for Lateral Route Optimization Opportunities}

This scenario illustrates how TAP may assist pilots in optimizing their route due to a weather update. First described in Ref 1, this scenario is an example of how TAP's automated monitoring can provide a lateral route change maneuver that would reduce flight time. In Figure 1, a TAP-equipped aircraft ("TAP Aircraft") had previously been

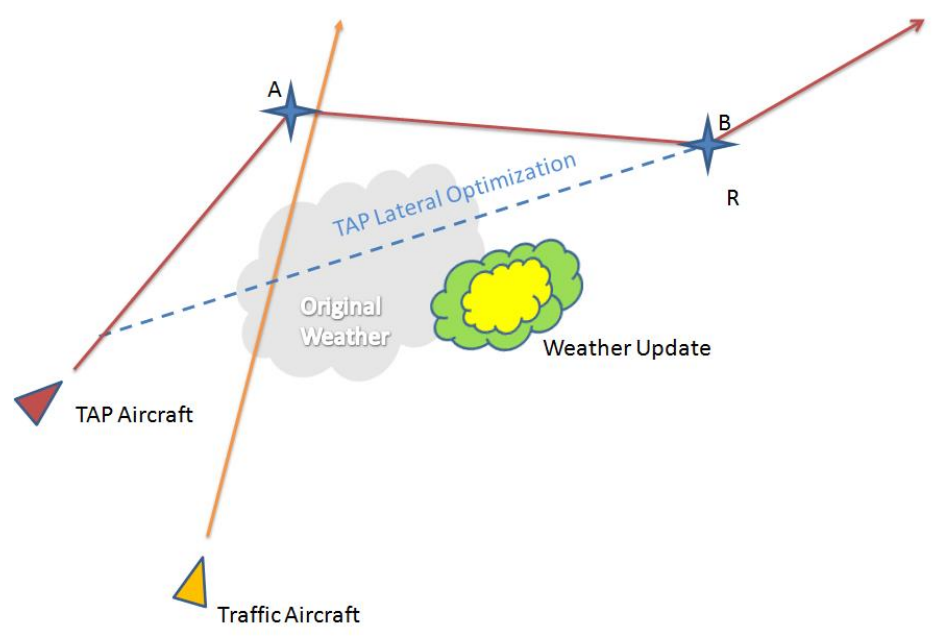

Figure 1 Lateral Route Change Optimization Scenario. 3

American Institute of Aeronautics and Astronautics 
redirected to Fix A to avoid convective weather ("Original Weather"). TAP has been set to automatically monitor for optimization opportunities that reduce flight time. It receives a weather update ("Weather Update") that indicates the convective weather system has dissipated. Due to this weather data update, TAP's optimization search algorithms generate a lateral route change (specifically, a direct to Fix B) that removes the original redirection around the weather. The algorithms assure that the route change accounts for separation from known nearby traffic ("Traffic Aircraft"). The TAP optimization solution ("TAP Lateral Optimization") also saves fuel, but is optimized to save time based on the pilot's optimization preference inputs. The pilot may choose to use this optimization solution to make the request to ATC using normal request procedures, and ATC reviews the request, as is done today.

\section{Automated Monitoring for Combo (Lateral / Vertical) Route Optimization Opportunities}

This scenario illustrates how TAP's auto monitoring for optimizations can provide route changes that may not be readily apparent to pilots. In Figure 2, a TAP-equipped aircraft ("TAP Aircraft") initially filed a flight plan with a cruise altitude that optimized for the wind data available at the time. Once airborne, the TAP system receives an update that indicates a wind field with more favorable winds at a different altitude than the aircraft's current cruise altitude ("Favorable Wind Altitude"). TAP's search algorithms, which in this example scenario are set to automatically monitor for optimization opportunities that reduce fuel consumed, generates a route change solution that combines both a vertical change to the more favorable altitude as well as a lateral change (to Fix C) to avoid creating a conflict with traffic ("Traffic Aircraft"). The pilot notes the suggested route change and that its outcomes indicate it will save a significant amount of fuel. The pilot may then choose to request the suggested route change from ATC, using normal procedures. ATC reviews the request, as is done today.

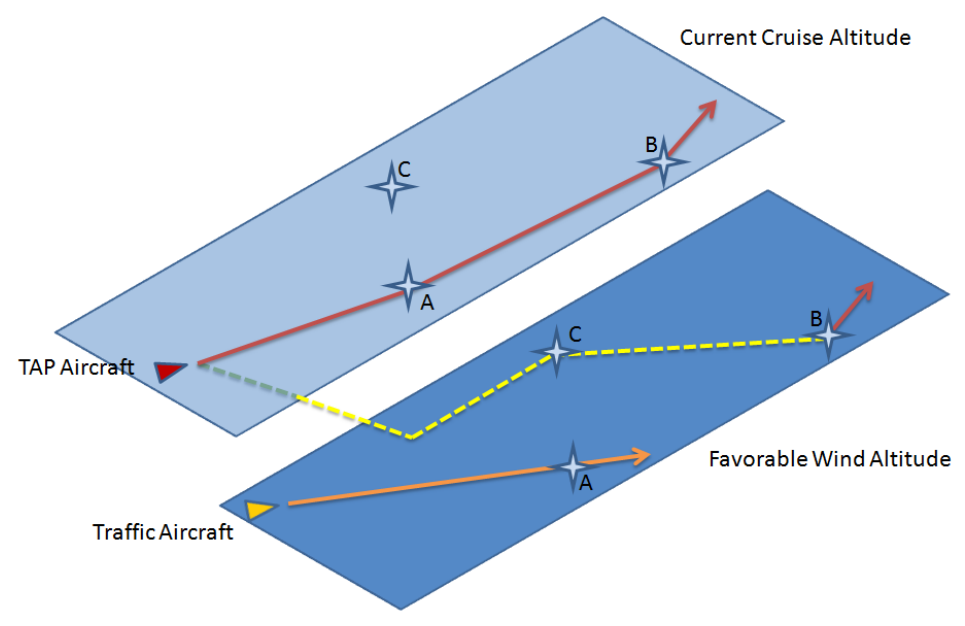

Figure 2 Combo Route Change Optimization Scenario.

\section{Manual Optimization}

In addition to providing automatic monitoring for route optimizations, TAP also provides tools for pilots to manually build conflict-free route changes. An example of such a scenario is shown in Figure 3.The crew of a TAPequipped aircraft ("TAP Aircraft") have received reports of turbulence ahead at their current cruise altitude, and that flight levels above and below them are smoother. Although TAP could be supplied predictions of turbulent airspace for its automated mode, this scenario assumes such data are not available other than through pilot reports on the radio. Initially, the crew manually enters the lower altitude into TAP ("Manual Route Change 1"). TAP indicates there is conflict with a convective weather system. The crew then manually enters the vertical change to the higher altitude ("Manual Route Change 2"). TAP evaluates the change and indicates the maneuver is conflict free, but that its outcome would consume a significant amount of fuel. The pilot then re-enters a maneuver to the lower altitude, but adds an off-route waypoint (Fix A) to re-direct around the weather system. TAP calculates that the route is conflict-free and displays this information to the pilot. The aircrew can than request the route change from ATC using existing procedures.

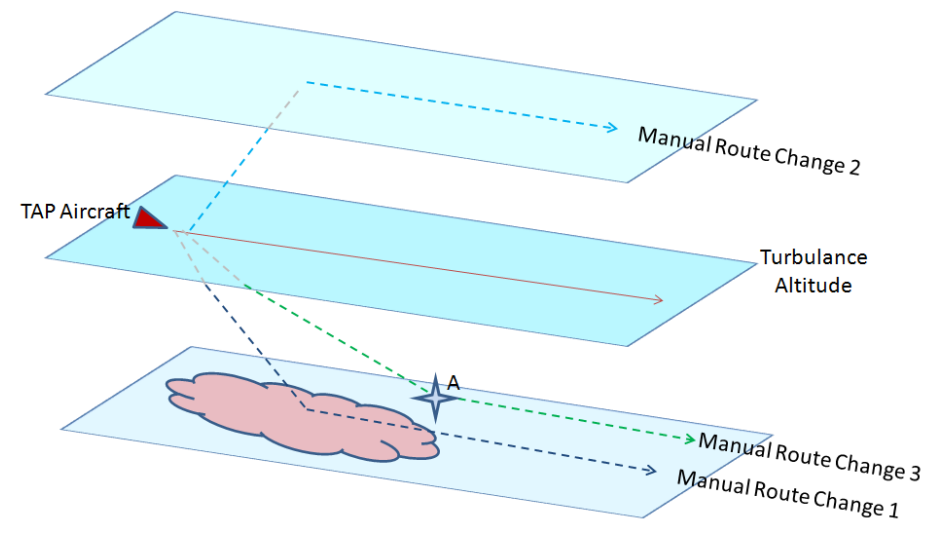

Figure 3 Manual Route Change Scenario. 


\section{Operational Environment}

TAP was developed to be used in current ADS-B equipped aircraft with minimal effect on cockpit configuration and data sharing capabilities. The following section details TAP's operating platform, data and configuration requirements, and assumptions of aircraft capability and conditions for operation.

\section{A. Platform}

In order to enable TAP to be available for near-term use without significant installation or certification costs, TAP was developed to operate on a Class 2 Electronic Flight Bag (EFB). ${ }^{3}$ Since this class of EFB provides "readonly" access to onboard avionics data, it allows for TAP to be installed without requiring any costly recertification of the existing avionics. Class 2 EFBs may receive data input from cockpit avionics via a data concentrator unit, the aircraft interface device (AID). The data concentrator reads data off of the cockpit avionics bus and sends it to the EFB using the ARINC 834-1 Simple Text Avionics Protocol (STAP). This read-only access to avionics data allows for TAP to be classified as a system that has minor or no effect to safety-critical operations, further streamlining the process of FAA approval. ${ }^{9,10}$

Since the Class 2 EFB is read-only, pilots will enter the TAP-proposed route changes into their FMS manually, as they do in current in-flight procedures.

For initial simulation and flight testing, TAP was installed on the Goodrich (now United Technologies Corporation) SmartDisplay ${ }^{\mathrm{TM}}$ Class 2 EFB configured to use the Windows XP operating system. Figure 4 shows TAP running on the SmartDisplay ${ }^{\mathrm{TM}}$ in the University of Iowa Operator Performance Lab flight simulator. The SmartDisplay ${ }^{\mathrm{TM}}$ EFB is currently in use by several airlines. As discussed in Section VI, versions of TAP that would operate on other platforms, such as Apple iPads, are currently being considered for future developments.

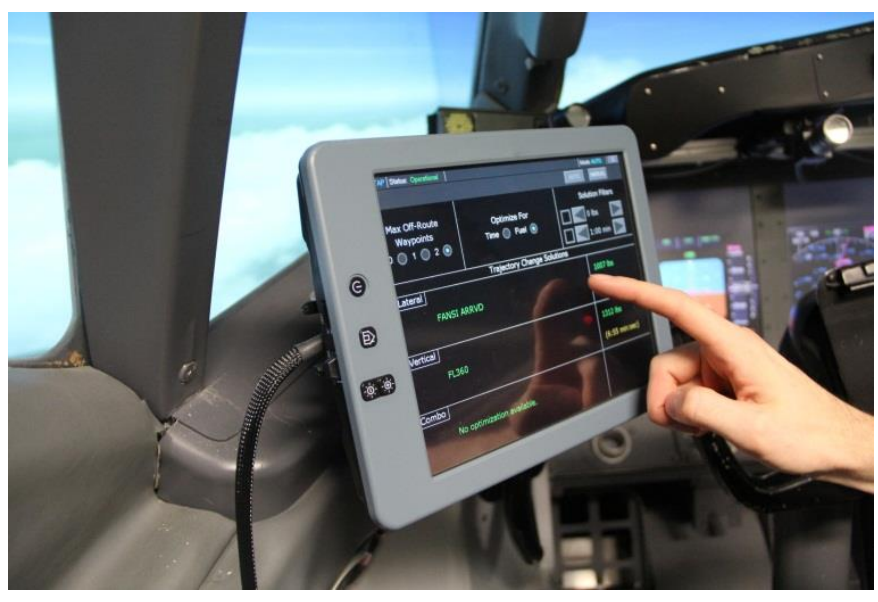

Figure 4 Class 2 EFB used for TAP prototype.

\section{B. Data Input Requirements}

TAP has been designed to receive data via both the STAP feed from the Class 2 EFB's data concentrator and from an External Data Server (EDS) that will provide data from other sources such as the internet or on-board satellite weather systems.

The avionics equipment that will provide specific data types via the STAP feed will differ depending on the specific cockpit configuration of the ownship aircraft, and not all data sources will be available in all environments. A configuration specification for each aircraft installation will map which avionics equipment will be providing specific data, (e.g., longitude and latitude, which may be available from more than one source) and which data concentrator ports need to be subscribed to for TAP to receive those data.

Sources of data via the EDS will also differ from one cockpit to another, and each aircraft will require a configuration specification defining what data TAP will expect via the EDS. The EDS itself will handle the processing of the data into TAP-specific format.

Ideally, a configuration of TAP will have a data source for the following:

1) Current Time

2) Ownship State and ID: including current position, altitude, true and magnetic track, vertical speed, Mach, calibrated airspeed, true airspeed, ground speed, and gross weight

3) Flight Control Computer Guidance Mode Settings

4) FMS Route Data: including active route waypoints, waypoint speed and time constraints (if any), cruise altitude, climb and descent speeds

5) Aircraft Performance Limits: including maximum altitude

6) Atmospheric Data: wind and temperature updates

7) Airspace Hazards: convective weather, restricted airspaces, and terrain (specified as polygons)

8) Traffic Hazards: current position and velocities and intent (if available) of nearby traffic 


\section{Navigation Database}

TAP provides route change requests using named waypoints to facilitate voice communications. To support this capability, TAP uses a navigation database of waypoints based on ARINC 424 that corresponds to the database used by the ownship aircraft's FMS. An adaptation process will assure that no waypoint suggested by TAP cannot be recognized by the FMS system or ATC when the pilot requests the proposed route change. It also assures that TAP will be able to recognize all the waypoints of the active route currently used by the FMS for guidance.

\section{Conditions for Operation}

TAP goes into operational mode when the ownship aircraft climbs above 10,000 feet (as specified in the TASAR operational concept, which focuses on en-route operations). Current TAP development has focused on its use during the en-route, cruise portion of the flight, but since it is built upon the AOP platform that is designed for all en route phases of flight, it is planned to extend TAP to the other phases of flight, as appropriate. Since opportunities for optimization diminish the closer an aircraft gets to its destination, the most optimal time to get the most benefit from TAP is the early stages of the en-route portion of the flight. ${ }^{4}$

\section{TAP Description}

The following sections will provide an overview of TAP's system architecture and its functional capabilities.

\section{A. TAP System Architecture}

The TAP system, the current version of which is intended to run on a Class 2 EFB, consists of three executable components: TAP Engine (processor), TAP Display (user interface) and External Data Server (EDS). All executables, configuration files, and output logs of the TAP System reside on the Class 2 EFB platform.

Figure 5 provides an overview of the TAP system architecture and how it accesses data. The Class 2 EFB will have a STAP-formatted data input feed from its data concentrator, which will provide the TAP Engine access to avionics data. The EDS, which provides formatted data to the TAP Engine from sources other than the avionics, will have access to the internet via the Class 2 EFB's Ethernet connection to the aircraft's broadband internet provider system. Other possible data sources, such as satellite weather systems ${ }^{3}$, could also be accessed by the EDS using the EFB's Ethernet connection as long as they are part of the aircraft's local area network.

NOTE: The EDS, which can provide data to multiple instances of TAP, could be run somewhere else in the cockpit environment, if desired, as long as it has Ethernet connectivity with the EFBs running TAP. Such an arrangement would minimize the use of the aircraft's broadband access, since only one EDS system would be downloading data via Data Sources

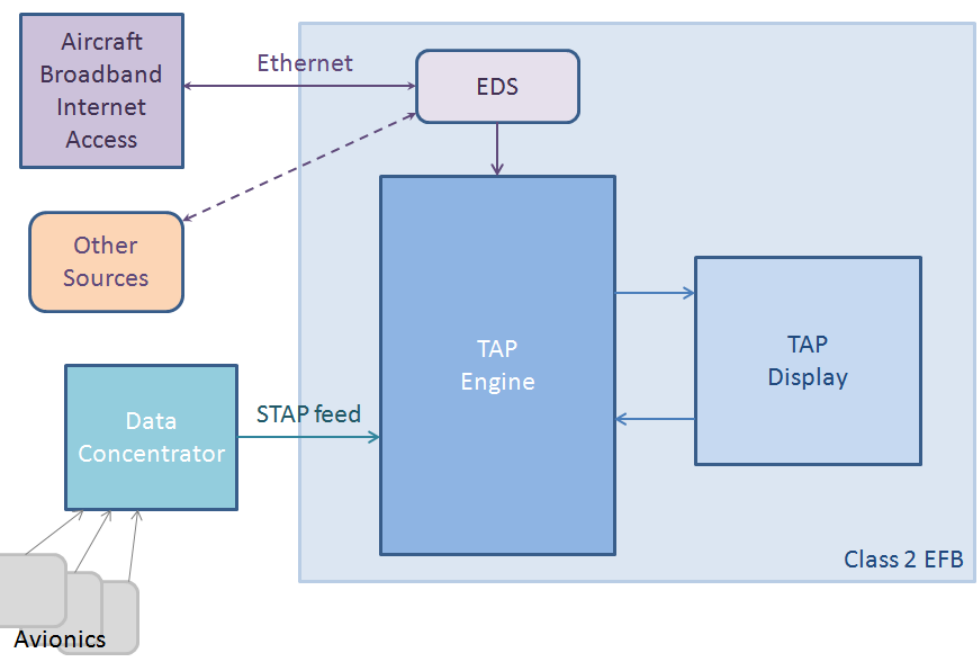

Figure 5 TAP system architecture. the internet.

\section{TAP Engine}

The TAP Engine, the main processor of the TAP system, is a specific configuration of AOP and therefore shares much of AOP's software architecture. The architecture, algorithms, and performance of AOP are described in detail in Refs 5-8. This section highlights some of the differences between TAP and AOP.

One difference is the TAP Engine's stand-alone operational mode. AOP was developed to operate as part of NASA Langley's Airspace and Traffic Operations Simulation (ATOS), and thus it was architected to synch with the simulation's mode controls. Since TAP is intended for use in various simulators and real aircraft, TAP Engine has been configured to be completely de-coupled from ATOS mode controls so that it can operate as a stand-alone system. 
Another difference is TAP's ability to read the STAP-formatted data input. In ATOS, AOP has direct access to ARINC 429-formatted avionics data via an Avionics Bus emulator in the simulation. This allowed AOP to access avionics data in a "report" form, or a complete time-stamped data set, from specific pieces of avionics equipment. In contrast, the STAP-format provides all data in atomic form, one datum at a time. Processing was added to the TAP Engine to handle data management of the STAP-feed and re-couple atomic data from specific data sources such that AOP's existing algorithms could work with the data without further adaptation.

Since AOP is a self-separation tool, its main functionality is to continuously probe for separation violations and generate optimized trajectory changes to avoid any detected conflicts. In contrast, TAP is specifically an optimization tool and is continuously probing for trajectory changes that optimize fuel or time without impacting traffic. This difference requires a more flexible capability to find the most optimal route change, while still accounting for traffic separation.

To perform conflict resolution, AOP uses a pattern-based genetic algorithm (PBGA) ${ }^{7}$ to generate a modification to the active route based on a predefined set of maneuver pattern templates (e.g., lateral offset, path stretch, direct intercept). TAP re-uses the PBGA, but its different function (optimization, rather than conflict resolution) requires maneuver pattern templates specific to the task of performing route optimization.

A new pattern was created for each of the two lateral types of TAP optimization that involve off-route waypoints: (1) a route change to one off-route waypoint and then reconnect to a downstream waypoint on the active route, and (2) a route change to two off-route waypoints and a reconnect. For vertical patterns (i.e., step climbs and descents) and a lateral direct to a downstream waypoint (direct-to-waypoint), there is only a finite set of possible solutions, and a genetic algorithm is not required. In these cases, TAP generates the complete set of possible routechange candidates and evaluates which is the best solution in flight time or fuel burn. For the direct-to-waypoint solution, this best solution will compete with the other pattern-generated solutions in generations of the genetic algorithm to determine the best overall lateral solution. The PBGA implementation for TAP is further described in Ref. 11.

Another added functionality of TAP beyond AOP capabilities is the generation of route changes using only named waypoints. Since AOP is directly integrated with the aircraft's FMS, off-route waypoints are generated in latitude and longitude coordinates and directly uploaded into the FMS for execution. TAP needs to generate route changes that may be easily communicated to ATC in a voice request and entered into the flight control avionics by hand. TAP Engine algorithms have been augmented to generate route changes that use only named waypoints from a navigation database, rather than latitude and longitude coordinates, though the latter option is still available for future enhanced versions of TAP. A comprehensive description of the new patterns and the process of generating route changes using named waypoints is in Ref 11.

\section{TAP Display}

The TAP Display is the Human Machine Interface (HMI) of the TAP system. The HMI was designed with guidance from current pilots and human factors experts to be:

- Easy to use on Class 2 EFB: The HMI design accounts for limitations of size, touch-screen only (no keyboard or mouse), and expected visibility issues of a Class 2 EFB mounted in an cockpit environment.

- An advisory-only tool: TAP should not distract the pilot from any safety-critical operations.

- Require minimal user commands: TAP should minimize user inputs to perform core functionality.

- Qualify as an EFB Type B software application: TAP has been designed to have no or minor impact on current safety critical operations, and it will not display ownship location or traffic positions on any of its displays.

- Communicate information clearly: TAP optimization and conflict information is displayed in a clear, easily deciphered manner that will allow pilots to quickly assess if an optimization solution is a candidate for an aircrew request.

\section{External Data Server (EDS)}

The External Data Server is a separate process that handles the connection, download, and processing of data from sources other than the ownship's avionics data via the STAP feed. Its initial version downloads winds and temperature aloft data from the Rapid Refresh service available via internet from the National Oceanic and Atmospheric Administration.

EDS was designed to be expandable to incorporate numerous other data sources as well. The adaptation of data sources will require analysis of the source data and what sub-set of the available data will be of use to a single 
instance of TAP, given its ownship location and active route. EDS connects to the data source, requests the data (or appropriate sub-set), converts it into TAP format, and passes it to the TAP Engine.

Currently, EDS is an on-board application only. Future enhancements of EDS include a proposal to introduce a ground-based server component to handle larger data management of internet sources, which the on-board version of EDS will communicate with to request a data sub-set appropriate for its ownship flight.

\section{B. TAP Functionality and Capabilities}

TAP has two main modes of operation: Auto Mode and Manual Mode. In Auto Mode, TAP continually monitors for optimization opportunities and generates conflict-free route change solutions that meet the aircrew's settings for objectives. In Manual Mode, TAP provides functionality to assist aircrews in manually creating route changes that will be acceptable to ATC as well as meet their objectives.

Outside of its two main operational modes, TAP includes an Initialization mode, which as part of the power-onsystem-test procedures on the tarmac before take-off, verifies that TAP has connectivity to all its required data sources. It also has a Standby mode, which TAP stays in until the operational conditions (complete data, aircraft above 10,000 ft) are met to go into one of the two operational modes. These screens are shown in Figure 6
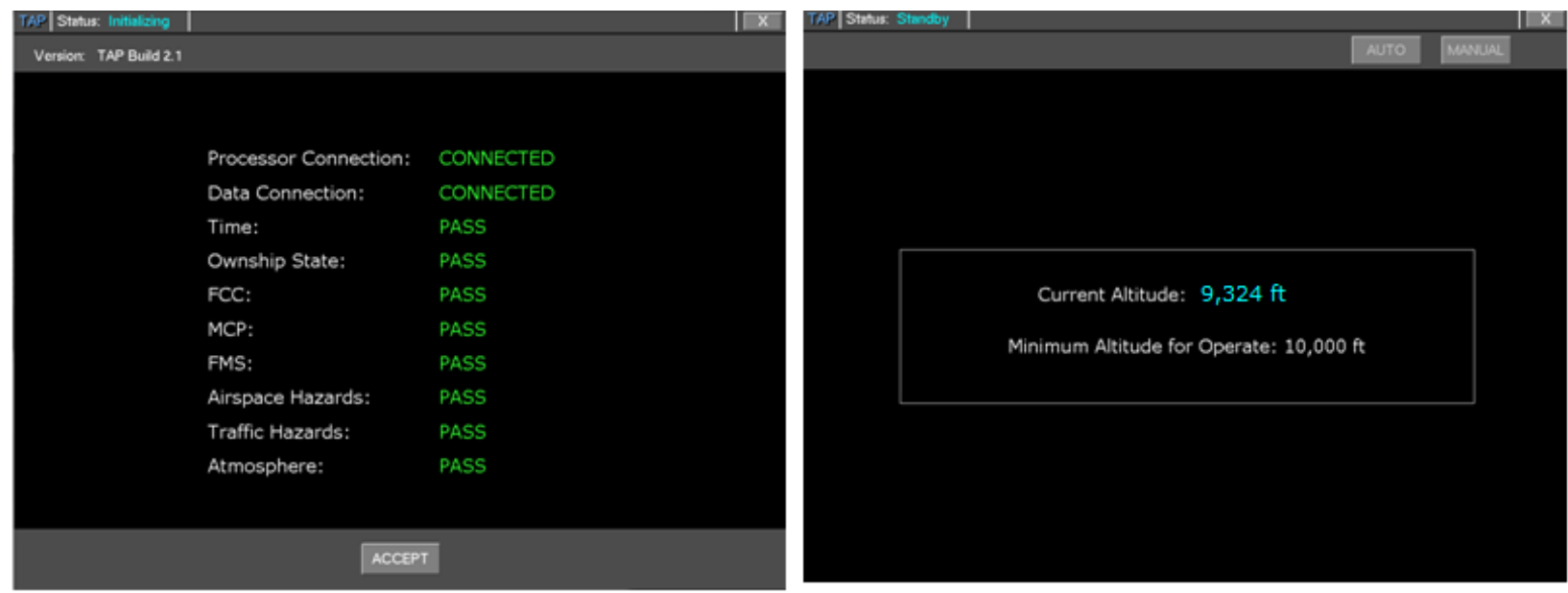

Figure 6 - TAP Initialization and Standby screens.

\section{Auto Mode}

When in Auto Mode, TAP continuously generates a set of optimizations at a one-minute update rate, giving the pilot a reasonable amount of time to determine whether they will pursue requesting the optimization from ATC. There are three types of optimizations TAP is capable of generating:

- Lateral: Lateral-only maneuver, with up to two waypoints off-route before reconnecting to the active route at a named waypoint. All waypoints are named waypoints that may be easily communicated verbally to ATC.

- Vertical: Cruise altitude change. All maneuvers are a VNAV climb or descent to a new cruise altitude.

- Combo: Maneuver that includes both a cruise altitude change and a lateral maneuver. This is a unique and independent solution, not just a composite of the lateral-only and vertical-only optimizations.

Pilots have the option to configure TAP Auto Mode to only display or generate optimizations that meet certain criteria. These configuration settings include:

- Maximum number of off-route waypoints $(0,1$, or 2$)$

- Specification of time or fuel optimization objective

- Filter specifying minimum fuel and/or time savings 
Figure 7 shows the main screen of TAP in Auto Mode. Since TAP Auto Mode is intended to be advisory-only and to run continuously without distracting the pilot, the HMI was designed to require no interaction from a user unless they want to change settings or if they want to select a specific optimization for further consideration.

TAP Auto Mode will search for trajectory optimizations once a minute. For each optimization solution, TAP calculates the predicted outcomes of the route change, i.e., the effect on fuel and flight time. These outcomes are refreshed every ten seconds.

Since the current version of TAP only optimizes for either time or fuel, it is possible for TAP to generate an optimization solution that saves time but costs fuel, or vise versa. For instance, in the example shown in Figure 7, TAP has been set to optimize for fuel rather than time. Both the vertical and combo solutions provide fuel savings, but actually add flight time. To indicate this in the Outcomes column, TAP displays the extra flight time in yellow and within parenthesis. This distinction allows aircrews to quickly assess the impact and acceptability of the trajectory change. Users may easily change the optimization goal of the Auto Mode using the "Optimize For" setting. When a new optimization goal is selected, a new trajectory optimization search is immediately initiated.

TAP's optimization algorithm will produce the best conflict-free optimization solutions it can find given the current traffic and area hazards. If no optimization solution of a certain type (e.g., Lateral) can be found, the solution for that generation cycle will read No Optimization Available. The algorithm will not return a solution with improved outcomes that conflicts with traffic or airspace hazards, nor will it return a solution with both outcomes negative unless airspace hazard locations dictate this as the best available option that avoids the airspace hazards.

If an aircrew desires to only see optimization solutions that save at least a certain amount of fuel and/or time, they may use the "Solution Filters" controls to set a minimum value for fuel and/or time saved. If TAP generates a solution that does not meet these minimum outcomes, the solution will read Solution Filtered and the Outcomes field will be blank. If the user wishes to see an optimization solution that has been filtered, they may use the "Solution Filters" controls to disable the filters (via checkboxes to the left of the filter values). With the filter off, the solution and its outcomes will be displayed.

Lateral and Combo solution types may have up to two off-route waypoints. If the user wishes only to see solutions that are direct reconnects (zero off-route waypoints) or have just one off-route waypoint, they may limit the number of off-route waypoints in the "Max Off-Route Waypoints" control section. Again, TAP will always generate the best solution possible within these constraints; if the maximum off-route waypoints is set to two offroute waypoints, but a direct-to-waypoint is the best conflict-free lateral solution, that is the solution that TAP will generate. An example of this can be seen in Figure 7. Maximum Off-Route Waypoints is currently set to two waypoints, but the lateral maneuver of both the Lateral and Combo solutions are a direct-to-waypoint to the waypoint SCRAN.

If the aircrew sees an optimization solution they feel may be a candidate for an aircrew request to ATC, they can "freeze" the optimization by selecting it on the Auto Mode main screen (i.e. by tapping the solution). Freezing an optimization prevents TAP from overwriting the selected optimization in the next automatic generation cycle.

When an optimization is selected, TAP Auto Mode switches to the Selected Optimization layout, as seen in Figure 8. Once selected, an optimization will remain frozen until the user cancels the selection without action (CANCEL) or records ATC's response to the resulting aircrew request (ATC ACCEPT or ATC DENIED). 


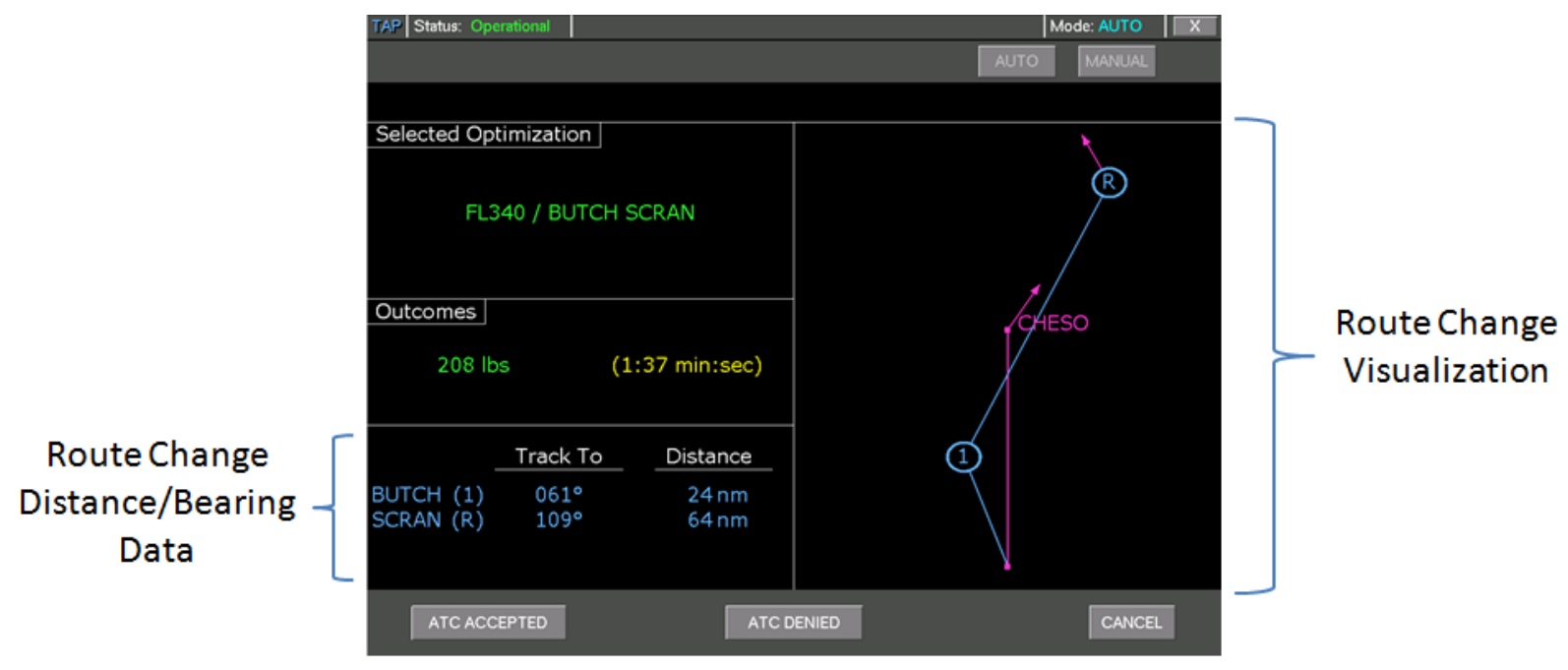

Figure 8 - Selected Optimization Screen.

TAP re-evaluates a selected optimization every ten seconds, checking if the route change is still conflict-free and if it is still considered reasonably navigable (i.e., lies within preset geometric ranges as described in Ref. 11). As an example of the latter case, a pilot selects an optimization and then gets called away from TAP without cancelling the frozen optimization. The aircraft continues to fly its active route and eventually passes the point where the initial turn of the route change becomes excessive. Once a selected optimization is no longer considered reasonably navigable, TAP turns the text of the selected optimization yellow, removes all route visualization and outcomes, and displays Unable to Evaluate Route Change at the top of the screen.

Each re-evaluation of the selected optimization will update the outcomes of the route change. This allows the pilots to monitor if the route change is still attractive as they consider whether to make an aircrew request to ATC. These outcomes will be generated as long as the route change is still considered reasonably navigable, and their color and format follow the same convention used on the Auto Mode main screen (i.e., green for savings, yellow with parentheses for cost).

If a re-evaluation of a selected optimization determines that the route change would conflict with a hazard, the text of the selected optimization will be turned yellow, and a message at the top of the screen will indicate the type of hazard. These conflict messages can have the following values:

- TRAFFIC

- WEATHER

- SUA

- TRAFFIC \& WEATHER
- $\quad$ TRAFFIC \& SUA

- WEATHER \& SUA

- $\quad$ TRAFFIC \& WEATHER \& SUA

For selected optimizations that include a lateral maneuver (Lateral and Combo), the Selected Optimization screen provides a graphical depiction of the route change relative to the active route ("Route Change Visualization" in Figure 8) that allows a pilot to quickly assess the geometric nature of the optimization. Pilots are unlikely to have knowledge of the relative location of every possible navigation fix relative to their present position. This display allows them to answer immediate questions such as whether the initial turn is right or left and how far the waypoints of the route change are relative to one another. A list view detailing the route change bearings and relative distances is also provided for clarification of the graphics and to assist with entry into the FMS and the ATC request.

This graphical display does not show current ownship position. It depicts the active route (magenta) from the point where TAP calculates the route change will diverge from the active route prior to the next active route waypoint (CHESO, in Fig. 8). The route change (shown in blue) is depicted relative to the current heading, including turns after off-route waypoints, and it indicates the nature of the turn at the reconnect point (Point R).

Once a pilot is satisfied that a selected optimization is acceptable (for which procedures may dictate entering the route change into the FMS and verifying acceptability using certified systems), they may proceed with communicating the route change request to ATC. When ATC response is received, the pilot selects ATC ACCEPT 
or ATC DENIED to record the outcome for later analysis, if desired. TAP will release the frozen optimization, return to the Auto Mode main page, and restart the search for new optimizations.

If the user wishes to release the frozen optimization without using it for an aircrew request, they may select CANCEL and return to the Auto Mode main screen.

\section{Manual Mode}

Manual Mode enables the pilot to directly enter a desired route change and have TAP evaluate it for fuel and time outcomes, as well as indicate if the route change is predicted to conflict with traffic or area hazards. This allows users to control the objective of the route change, which may be something other than fuel and time savings, such as avoiding turbulence reported ahead at the current cruise altitude. If TAP detects that there is a conflict in a manually entered route change, it allows the user to alter the route change and re-evaluate.

Manual Mode accepts route changes of the same three types generated in Auto Mode:

- Lateral: Lateral-only maneuver which may include up to two off-route named waypoints and a reconnect with the active route at a named waypoint.

- Vertical: Cruise altitude change

- Combo: Maneuver that includes both a cruise altitude change and a lateral maneuver

There are two main parts to Manual Mode's functionality: 1) Allowing users to manually enter a route change, using named waypoints for both reconnect and off-route waypoints, and 2) Evaluating the route change for conflicts and fuel and time outcomes.

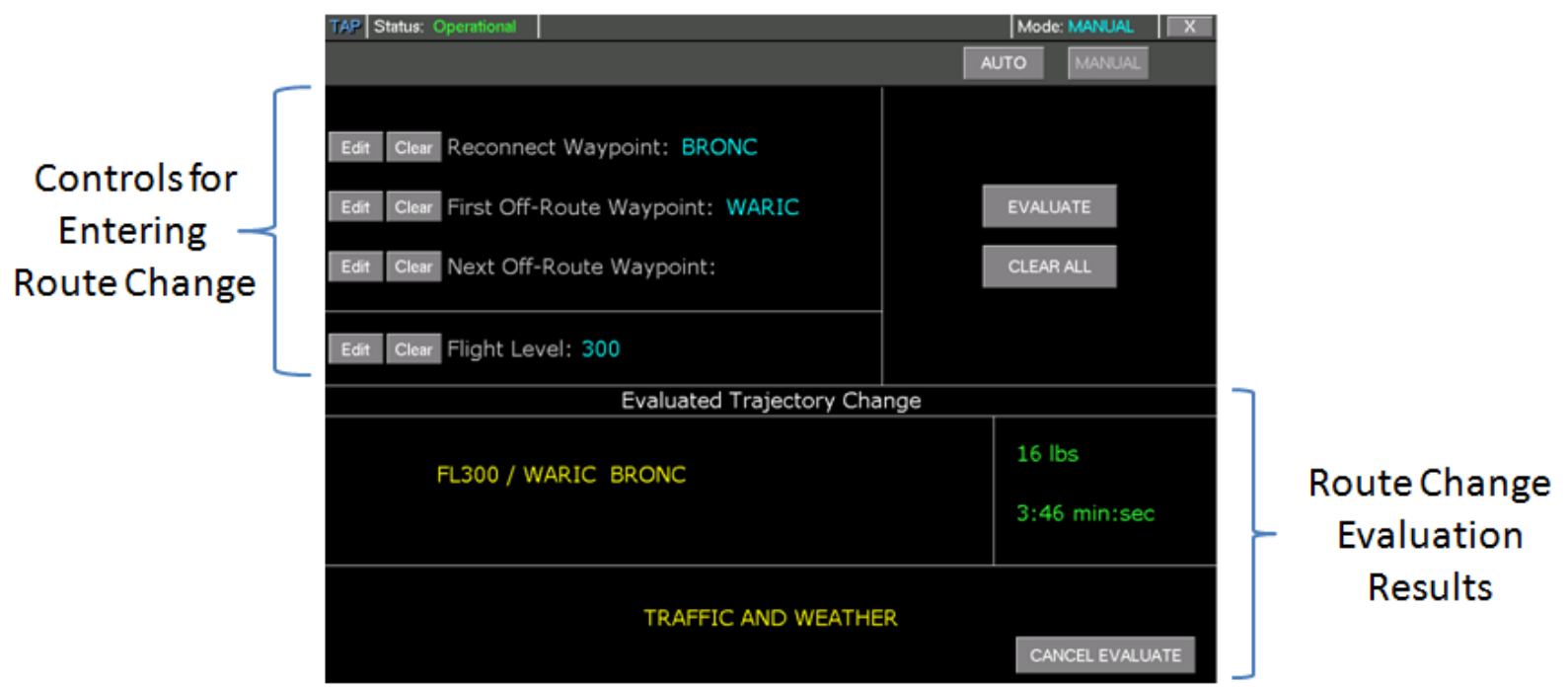

Figure 9 TAP Manual Mode Main Screen.

Figure 9 shows an example of the Manual Mode's main screen. The top section provides the controls for entering a route change. The lower portion of the screen shows the results of TAP's evaluation of a route change. The example shown includes a new cruise altitude (FL300) combined with a lateral maneuver consisting of one offroute waypoint (WARIC) and reconnecting with the active route at BRONC. TAP's evaluation shows that the proposed route change would save both fuel and flight time, but also that it would conflict with both traffic and weather hazards. Because a conflict is predicted, the evaluated route change is displayed in yellow text, indicating that it is less likely to be approved by ATC. If the evaluation had not found a conflict, the text would be green.

TAP refreshes the evaluation of the manual route change every ten seconds. The refresh updates the outcomes, and it rechecks if the route change is still conflict-free and considered reasonably navigable. A user may leave a manual evaluation displayed as long as they wish, but if it is no longer considered reasonably navigable, no outcomes are calculated.

To define the route change manually, TAP provides several controls to expedite the process for the pilot. As stated earlier, the pilot may define lateral-only, vertical-only, or combo route changes. TAP will not allow a route change to be submitted for evaluation until it meets the following criteria:

- Lateral maneuvers (if any) include specification of a reconnect waypoint on the active route

- Vertical maneuvers (if any) include a new flight level other than the current cruise altitude 
To assure that the pilot only designates a reconnect waypoint that is on the active route, TAP provides a drop-down list of the existing route's waypoints, as shown in Error! Reference source not found.. If this reconnect point is subsequently removed from the FMS active route while the TAP Manual Mode screen is active, TAP's evaluation will indicate that TAP is no longer able to evaluate the route.

When designating off-route waypoints, the pilot may not necessarily know the names of the waypoints that meet their current desires for a route change. For instance, the aircraft may be near scattered convective weather, and the pilot may be considering a request to deviate left 10 degrees. TAP provides a tool to allow a pilot to request the nearest waypoint found near a given bearing and distance relative to the ownship's state. The pilot may enter the relative bearing of the initial turn they desire, followed by the desired distance on that track, and then instruct TAP to search for a waypoint. In Error! Reference source not found., the pilot is searching for an off-route waypoint $100 \mathrm{~nm}$ and 10 degrees to the left of their present location. TAP has found JASPE to be closest to this point, and it provides the actual bearing and distance to JASPE. TAP refreshes the search for the waypoint every ten seconds to account for the ownship's change of location. The pilot may use the SELECT button to insert the identified waypoint as the off-route waypoint or may cancel the waypoint editing process with the CANCEL button.

When a pilot is constructing a route change with two off-route waypoints, the same tool may be used to search for a second waypoint. The bearing and distance entered and displayed will be relative to the location of the first offroute waypoint, rather than the ownship location.

If the pilot knows the name of the waypoint they wish to use for an off-route waypoint, TAP provides an on-screen keyboard to allow the pilot to enter the name manually, as shown in Error! Reference source not found.. If the pilot enters a waypoint name that does not exist in the navigation database, TAP will inform the user that it cannot evaluate the manual route change due to the

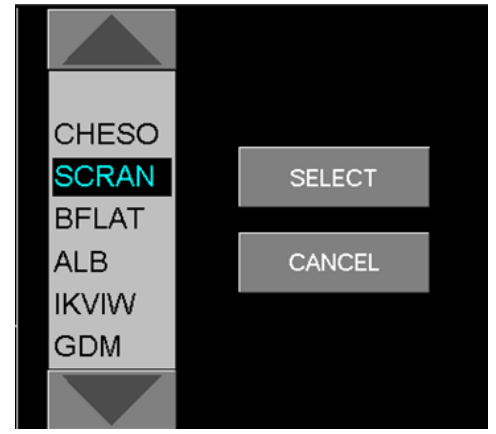

Figure 10 Reconnect waypoint scroll list.

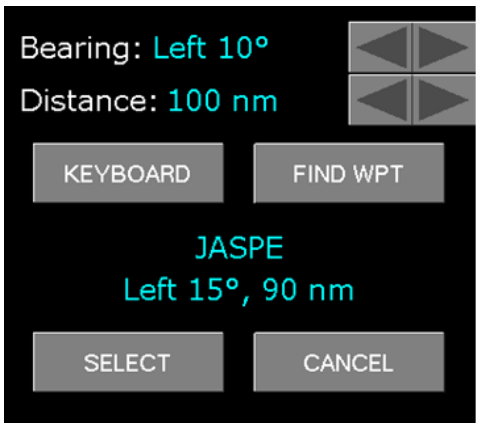

Figure 11 Waypoint search feature uses relative bearing and distance. invalid name.

To provide quick selection of a new cruise flight level, TAP provides a scrollable list of flight levels near the current altitude as shown in Figure 13.

The controls for editing a route change remain accessible while a route change is being evaluated. This allows a pilot to edit a route change based on the evaluation results as they are dynamically updated.

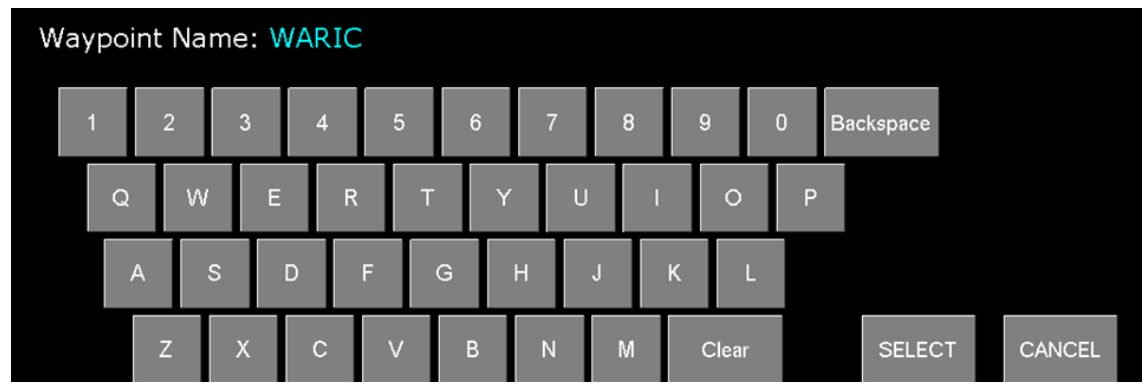

Figure 12 Keyboard input tool for specifying known waypoints.

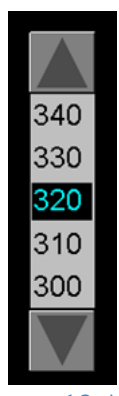

Figure 13 Altitude selector scroll list.

\section{Future Developments}

Several areas of further development have been proposed for TAP. This section summarizes some of these developments.

\section{A. Adapting TAP to run on other Class 2 EFB Platforms}


Feedback from the operator community has indicated a preference to install TAP on the EFB platforms already in use in their cockpits. The initial version of TAP's software is cross-platform and may run on Windows and UNIX-based operating systems. An analysis of adapting TAP to run on iOS (for Class 2 iPad EFBs) is in progress.

\section{B. Offer TAP Processing Functionality as an API}

Tools for flight planning have already been developed for EFBs that have very mature, customer-tested and comprehensive designs. Rather than require users to run TAP as a standalone system to gain its optimization capabilities, the development of an application programming interface (API) for the TAP processor would allow existing systems to augment their tools with TAP's optimization capabilities.

\section{Manual Mode Resolution}

When the evaluation of a manually entered route change in TAP's Manual Mode is found to have a conflict with traffic or area hazards, it is up to the pilot to edit the route change to resolve the conflict. Manual Resolution would provide the user the ability to let TAP provide an alternative, conflict-free route change that is as close as possible to the original intent of the manually entered route change.

\section{Airspace Boundary Awareness}

TAP's goal is to provide route change optimizations that are more likely to be approved by ATC. However, if a route change involves multiple re-crossings of an airspace sector boundary, ATC may not be inclined to grant the route change. Incorporation of knowledge of airspace boundaries into TAP's search algorithms can allow TAP to bias away from choosing such a route change. Additionally, TAP could determine the aircraft's proximity to the next sector and indicate to the aircrew whether they are likely in handoff status between sectors. The aircrew could then hold their request until checking in with the next sector controller.

\section{Conclusion}

The TAP prototype system provides the capabilities and functionality for initial testing and preliminary implementation of the TASAR concept, using existing architecture and algorithms from AOP, a self-separation research system that has been well-tested over the past decade. In the summer and fall of 2013, it will be tested in a HITL simulation at the University of Iowa Operator Performance Lab and a flight test in a Piaggio P180 Avanti aircraft. Results from these tests will provide insight on TAP's usability and will identify potential improvements to TAP's functionality.

Development of the TAP system has also provided improvements to the capabilities of the AOP system, which now has the ability to run in stand-alone mode and has been augmented with PBGA search patterns that expand its capabilities to perform optimization.

It is expected that TAP will continue to be enhanced further to meet the needs of users adopting TASAR for use in the NAS, as well as to provide enhanced optimization capabilities.

\section{Acknowledgments}

This development project was funded by NASA under contract NNL12AA06C. Development of the optimization and new PBGA patterns was performed by Dr. David A. Karr and Stephen DePascale of Engility Corporation. John Maris and Marie-Hélène Larose of Advanced Aerospace Solutions provided technical support in adapting TAP to operate on the Piaggio P180 Avanti test aircraft. Kelly Burke of NASA Langley provided humanfactors analysis of the prototype's HMI design. Matthew Cover and Nick Anderson of University of Iowa Operator Performance Laboratory provided the photo in Figure 4.

\section{References}

${ }^{1}$ Ballin, M.G. and Wing, D.J., "Traffic Aware Strategic Aircrew Requests (TASAR)", AIAA-2012-5623, AIAA $12^{\text {th }}$ Aircraft Technology, Integration, and Operations Conference (ATIO), Indianapolis, IN, USA September 2012.

${ }^{2}$ Henderson, J., Traffic Aware Strategic Aircrew Requests (TASAR) Concept of Operations, NASA/ CR-2013218001, May 2013.

${ }^{3}$ Wing, D. J., Ballin M., Koczo, S., and Vivona, R. A., "Developing an On-Board Traffic-Aware Flight Optimization Capability for Near-Term Low-Cost Implementation", accepted for publication, AIAA $13^{\text {th }}$ Aviation Technology, Integration, and Operations (ATIO) Conference, Los Angeles, CA, USA, August 2013. 
${ }^{4}$ Henderson, J., Idris H., and Wing, D.J., "Preliminary Benefits Assessment of Traffic Aware Strategic Aircrew Requests (TASAR)", AIAA-2012-5684, AIAA 12 th Aircraft Technology, Integration, and Operations Conference (ATIO), Indianapolis, IN, USA, September 2012.

${ }^{5}$ Karr, D.A., Vivona, R.A., Roscoe, D.A., and Wing, D.J., "Autonomous Operations Planner: A Flexible Platform for Research in Flight-Deck Support for Airborne Self-Separation", AIAA-2012-5417, AIAA 12 ${ }^{\text {th }}$ Aircraft Technology, Integration, and Operations Conference (ATIO), Indianapolis, IN, USA, September 2012.

${ }^{6}$ Karr, D. and Vivona, R., "Conflict Detection Using Variable 4D Uncertainty Bounds to Control Missed Alerts", AIAA-2006-6057, AIAA Guidance, Navigation and Control Conference, Keystone, CO, USA, 2006.

${ }^{7}$ Vivona, R., Karr, D., Roscoe, D., "Pattern-Based Genetic Algorithm for Airborne Conflict Resolution", AIAA2006-6060, AIAA Guidance, Navigation and Control Conference, Keystone, CO, USA, 2006.

${ }^{8}$ Karr, D., Vivona, R., Roscoe, D., DePascale, S., and Consiglio, M., "Experimental Performance of a Genetic Algorithm for Airborne Strategic Conflict Resolution", AIAA Guidance, Navigation and Control Conference, Chicago, IL, USA, 2009.

${ }^{9}$ Koczo, S. "Analysis of Operational Hazards and Safety Requirements for Traffic Aware Strategic Aircrew Requests (TASAR)”, NASA/CR-2013-218002, May 2013.

${ }^{10}$ Koczo, S. and Wing, D., "An Operational Safety and Certificaiton Assessment of a TASAR EFB Application", accepted for publication, $32^{\text {nd }}$ Digital Avionics Systems Conference, Syracuse, NY, USA, October 2013.

${ }^{11}$ Karr, D.A., Vivona, R.A., and Wing, D.J., "Costs of Limiting Route Optimization to Published Waypoints in the Traffic Aware Planner", AIAA Guidance, Navigation and Control Conference, Boston, MA, USA, August 2013. 